\title{
Task Partitioning in a Ponerine Ant
}

\author{
Guy Theraulaz*†, Eric Bonabeau $\$ \S$, RicardV. Solé $\|$, Bertrand Schatz* \\ and Jean-Louis Deneubourg
}

\begin{abstract}
*Laboratoire d'Ethologie et Cognition Animale, CNRS - ERS 2382, Université Paul Sabatier, 118 route de Narbonne, 31062 Toulouse Cédex, France $\ddagger$ EuroBios, 9, rue de Grenelle, 75007 Paris, France §Santa Fe Institute, 1399 Hyde Park Road, Santa Fe, NM 87501, USA \|Complex Systems Research Group, Department of Physics, FEN-UPC, Campus Nord B4, 08034 Barcelona, Spain $\uparrow$ Center for Nonlinear Phenomena and Complex Systems, Université Libre de Bruxelles, C.P. 231, Campus Plaine, B-1050 Brussels, Belgium
\end{abstract}

(Received on 11 July 2001, Accepted in revised form on 13 November 2001)

\begin{abstract}
This paper reports a study of the task partitioning observed in the ponerine ant Ectatomma ruidum, where prey-foraging behaviour can be subdivided into two categories: stinging and transporting. Stingers kill live prey and transporters carry prey corpses back to the nest. Stinging and transporting behaviours are released by certain stimuli through response thresholds; the respective stimuli for stinging and transporting appear to be the number of live prey and the number of prey corpses. A response threshold model, the parameters of which are all measured empirically, reproduces a set of non-trivial colony-level dynamical patterns observed in the experiments. This combination of modelling and empirical work connects explicitly the level of individual behaviour with colony-level patterns of work organization.
\end{abstract}

(C) 2002 Elsevier Science Limited. All rights reserved.

\section{Introduction}

Understanding the regulation of division of labour in insect colonies is essential for understanding the evolution of social behaviour (Oster \& Wilson, 1978; Bourke \& Franks, 1995). Division of labour refers to the division of the workforce among the range of tasks performed in the colony. Recently, attention has been directed towards the way in which workers actually performed a given task. The so-called task partitioning, whereby a piece of work is divided among two or more workers - such as the partitioning of the collection of a load of

\footnotetext{
$\dagger$ Author to whom correspondence should be addressed. E-mail: theraula@cict.fr
}

forage between a forager and a storer or a transporter - has been recognized as an important form of work organization in social insects (Jeanne, 1986; Ratnieks \& Anderson, 1999). Task partitioning may also exist in the absence of division of labour as it was pointed out by Ratnieks \& Anderson (1999; see also Anderson et al., 2001). What is particularly interesting about task partitioning is that it appears to require a higher degree of coordination and perhaps more complex exchanges of information among nestmates than other patterns of division of labour.

In a previous paper (Bonabeau et al., 1998), we have conjectured that a simple response threshold model (Robinson, 1987, 1992; 
Plowright \& Plowright, 1988; Bonabeau et al., 1996, 1998) could account for task partitioning when the stimulus for subtask 2 (for example, storing a load of forage) increases as a result of workers performing subtask 1 (for example, foraging). The idea behind the response threshold model is simple: when the intensity of a stimulus associated with a task exceeds the response threshold of a worker, that worker engages in task performance with a high probability in response to the stimulus. Task performance reduces the intensity of the stimulus, thereby decreasing the probability that other workers engage in the same task. If performing subtask 1 not only decreases stimulus intensity for subtask 1 but also increases stimulus intensity for subtask 2 , what should be observed is a form of task partitioning where the number of workers performing subtask 2 increases as subtask 1 is being accomplished. This prediction is yet to be tested in three important ways:

(1) identify a species in which such a phenomenon is observed,

(2) show the existence of a response threshold mechanism involved in the phenomenon, and

(3) measure empirically the model's parameters. Validating this model would clearly establish for the first time the connection between individual behaviour and coordinated colonylevel behaviour in the context of the regulation of division of labour.

The present paper is aimed at showing that the model does explain a pattern of task partitioning among hunters of the neotropical ponerine ant Ectatomma ruidum. In this species, hunting behaviour can be subdivided into two categories: stinging, that is, killing live prey, and transporting, that is, carrying prey corpses back to the nest (Schatz et al., 1996; Schatz, 1997). Such a behaviour of partitioned stinging and transporting is also observed in Pachycondyla caffraria (Agbogba \& Howse, 1992). We present a strong evidence that the stinging and transporting behaviours are released by certain stimuli through response thresholds; the respective stimuli for stinging and transporting appear to be the number of live prey and the number of prey corpses. In addition, we have been able to measure experimentally all the model's parameters. When the empirical values of the model's parameters are used, the model reproduces the various dynamical patterns observed in the experiments with a remarkable accuracy.

Section 2 introduces the experimental observations. The model is described in more mathematical detail in Section 3. The additional experimental measurements suggested by the model are described in Section 4. The results of simulating the model with empirical parameter values are given in Section 5. Finally, the evolutionary implications, the limitations, and the possible extensions of the model are discussed in Section 6.

\section{Experiments}

\subsection{METHODS}

Ectatomma ruidum is common in coffee or cocoa plantations where it preys upon a wide variety of arthropods (Lachaud, 1990). Colonies of E. ruidum, collected in the Mexican state of Chiapas, were reared in plaster nests and placed in an experimental room under controlled conditions (temperature: $25 \pm 1{ }^{\circ} \mathrm{C}$; humidity: $60 \pm 5 \%$; photoperiod: $12: 12 \mathrm{~L} / \mathrm{D}$ ). Fruit flies (Drosophila melanogaster) were offered to the colony as prey. The experimental set-up was composed of a foraging area $(30 \times 30 \mathrm{~cm})$ connected at one end with the nest and at the other end with the hunting area consisting of a circular Petri dish (diameter: $9 \mathrm{~cm}$, height: $1 \mathrm{~cm}$ ). The prey were provided in the hunting area. Four colonies were studied with five different quantities of prey (colony with 60 workers: 50, 80, 120, 150, and 200 prey; colony with 130 workers: $80,150,200$, 250, and 300 prey; colony with 240 workers: 80 , 150, 250, 400, and 500 prey; colony with 350 workers: 100, 200, 300, 400, and 500 prey). During preliminary observations, all workers performing any kind of predatory act were marked individually. During the experiments, all the behavioural acts of all the marked ants were recorded every $10 \mathrm{~min}$ over a 2 -h period.

\subsection{RESULTS}

As has been previously reported (Schatz et al., 1996; Schatz, 1997), two main types of behaviour 
can be clearly distinguished in E. ruidum hunters: stinging live prey and transporting dead prey. The typical sequence of attack of a stinger has been described by Lachaud (1990) and Schatz et al. (1997). Once the prey is motionless it is most often dropped on the ground. Dead prey lying on the ground are picked up by transporters and taken to the nest. Active solicitation of a stinger by a transporter for prey transfer may also be observed. Transporters usually come back to the hunting area. Workers can exhibit both behaviours within an experiment. Figure 1 shows the number of live and dead prey as a function of time as well as the fraction of workers observed in either the stinger or the transporter state, for three experimental situations which are representative of the main, observed dynamical patterns [130 workers and 80 prey (12 hunters involved), 130 workers and 250 prey (15 hunters involved), 240 workers and 500 prey (22 workers involved)]. The dynamical patterns can be subdivided into two main categories: those where the initial number of live drosophilas is relatively small (of the order of $50-80$ ) and those where the initial number of live drosophilas is relatively large (up to 500). In the first case [Fig. 1(a), (b)], the fraction of stingers first increases and then rapidly decreases, the fraction of transporters increases with a time delay with respect to the fraction of stingers, and then decreases slowly; the number of prey decreases quickly, while the number of corpses available for transport first increases and then decreases, but never becomes large. By contrast, in the second case [Figs. 1(c), (d) and (e), (f)], the fraction of stingers increases and reaches a stable plateau, the fraction of transporters increases with a small time delay and reaches a similar plateau; the number of live prey decreases steadily with time and the number of corpses in the arena increases steadily.

\section{The Model}

The idea behind the model (Bonabeau et al., 1998) is simple. When live drosophila are
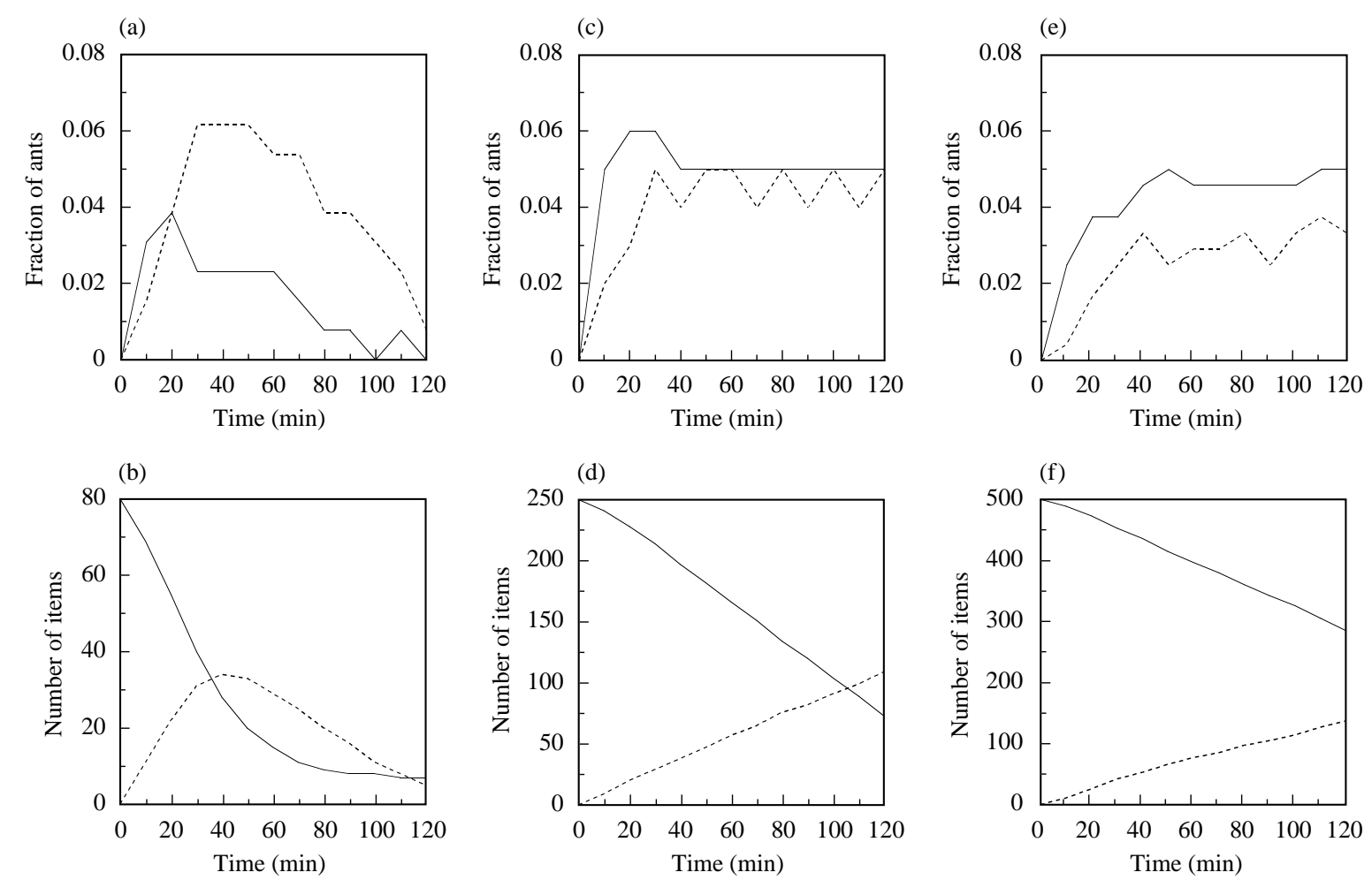

FIG. 1. Fractions of colony workers that are stingers or transporters, and numbers of live prey and dead bodies as a function of time for three different colony sizes and initial number of prey. $(a, b)$ Colony size $=130$, number of prey $=80$. $(c, d)$ Colony size $=130$, number of prey $=250$. (e, f) Colony size $=240$, number of prey $=500$. (a, c, e) $(-)$ stingers; $(\cdots)$ transporters. (b, d, f) (-) live prey; (------) dead bodies. 
presented to the colony, stinger behaviour is activated because the number of live prey exceeds the stinging threshold of workers. Workers start killing the prey, which leads to the presence of an increasing number of corpses to be transported to the nest. Transporter behaviour is stimulated as the number of corpses exceeds the transporting threshold of workers. This may explain the apparent task succession observed in the experiments with a small number of prey [Fig. 1(a), (b)]. When there is a large number of prey, the killing rate is not sufficient to take the stimulus below the stinging threshold of workers within the time frame of the experiment, so that the number of stingers is not observed to decrease (Figs. 1(c), (d) and (e), (f)].

Figure 2 gives a logical sketch of the model. Let $S$ be the total number of workers and $N$ the total number of potential hunters, that is, the equivalent of the number of workers that exhibits a hunting behaviour (stinging and/or prey transportation) at least once during an experiment. We assume that $N$ is given: determining how this number varies as a function of colony size requires another model. Let $x_{i}$ be the fraction of these $N$ workers engaged in performing task $i$ ( $i=0$ : non-hunting activity; $i=1$ : stinging, $i=2$ : transporting), $n_{1}$ the number of live prey in the hunting area, $n_{2}$ the number of prey corpses in the hunting area, $\tau_{i}$ the average time spent performing hunting task $i$ before

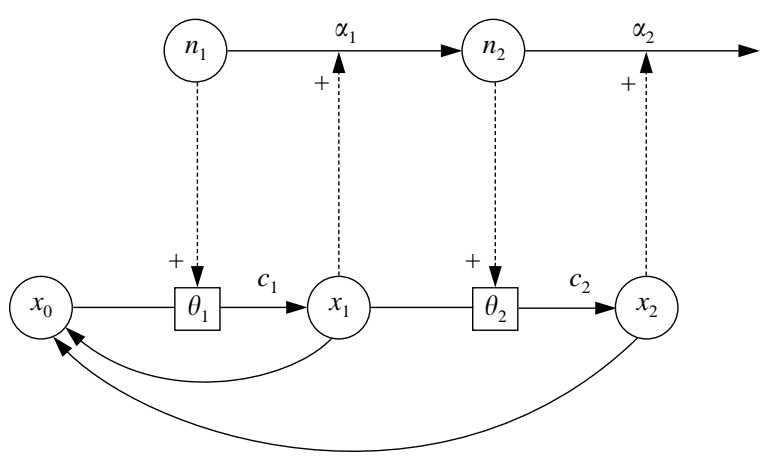

FIG. 2. Transitions between different states in the taskpartitioning model. Two basic "compartments" are defined: variables including stimuli $\left(n_{1}\right.$ : number of live prey; $n_{2}$ : corpses) and tasks $\left(x_{1}, x_{2}\right)$, with $x_{0}=1-x_{1}-x_{2}$ (see text). $(\longrightarrow)$ arrows indicate transitions in population values and (-.-.) arrows indicate interactions that allow transitions to occur. The square boxes introduce the presence of threshold functions weighting the effect of interactions. switching to a non-hunting task or before becoming inactive, $\alpha_{1}$ the efficiency of task 1 performance, $\alpha_{2}$ the efficiency of task 2 performance. $\alpha_{1}$ is also the rate of corpse production per stinger per time unit: the rate of increase of $n_{2}$ is proportional to $\alpha_{1}$. Finally, let $f_{1}=N x_{1} / S$ be the fraction of workers in the colony that are in the stinger state and $f_{2}=N x_{2} / S$ the fraction of workers in the colony that are in the transporter state.

The response threshold model (Bonabeau et al., 1996, 1998) assumes that the probability that a worker responds to the perceived presence of $n_{1}$ live prey by becoming a stinger is equal to $c_{1}\left(1-10^{-n_{1} / \theta_{1}}\right)$ where $\theta_{1}$ is the worker's response threshold associated with the stinging task and $c_{1}$ is a positive rate: in other words, when $n_{1} \gg \theta_{1}$, the worker is more likely to respond. Similarly, the probability that a worker responds to the presence of $n_{2}$ prey corpses in the hunting area by becoming a transporter is equal to $c_{2}\left(1-10^{-n_{2} / \theta_{2}}\right)$, where $\theta_{2}$ is the response threshold associated with the transporting task and $c_{2}$ is a positive rate. In reality, the stimuli that potential stingers or transporters respond to are certainly more complex but they can be abstracted away and included into the variables $n_{1}$ and $n_{2}$ as a first approximation. The values of $c_{1}$, $\theta_{1}, \tau_{1}, \alpha_{1}, c_{2}, \theta_{2}, \tau_{2}$, and $\alpha_{2}$ need to be estimated experimentally. The dynamics of $x_{1}, x_{2}, n_{1}$ and $n_{2}$ are described by

$$
\begin{gathered}
\partial_{t} x_{1}=c_{1}\left(1-10^{-n_{1} / \theta_{1}}\right)\left(1-x_{1}-x_{2}\right)-\frac{x_{1}}{\tau_{1}}, \\
\partial_{t} x_{2}=c_{2}\left(1-10^{-n_{2} / \theta_{2}}\right)\left(1-x_{1}-x_{2}\right)-\frac{x_{2}}{\tau_{2}}, \\
\partial_{t} n_{1}=-\alpha_{1} N x_{1}, \\
\partial_{t} n_{2}=\alpha_{1} N x_{1}-\alpha_{2} N x_{2},
\end{gathered}
$$

where $\partial_{t}$ denotes derivatives with respect to time. The first right-hand term of eqn (1) expresses that new stingers are "recruited" from the fraction $\left(1-x_{1}-x_{2}\right)$ of potential hunters which are not yet stingers or transporters, and that such workers are recruited with probability $c_{1}\left(1-10^{-n_{1} / \theta_{1}}\right)$ per time unit. The second 
right-hand term of eqn (1) reflects the fact that stingers give up hunting after spending a time $\tau_{1}$ on average in the stinger state. Equation (2), which describes the dynamics of the fraction of transporters, $x_{2}$, is very similar in structure and meaning to eqn (1). Notice that no direct transition from stinging to transporting or from transporting to stinging is included in the equations; individuals can, and are observed to, perform the two tasks within an experiment, but this requires a transition to the non-hunter state first. Equation (3) states that the number of live prey, in the absence of an inflow of such a prey into the arena, decreases at a rate $\alpha N x_{1}$ : if there are $N x_{1}$ stingers within a time unit $\left(x_{1}\right.$ is the fraction of stingers and $N$ the number of potential hunters, so that $N x_{1}$ is the total number of stingers), each killing $\alpha_{1}$ prey within that time unit, then a total of $\alpha_{1} N x_{1}$ prey is killed during that time unit. Equation (4) states that the $\alpha_{1} N x_{1}$ killed prey become corpses that can be transported to the nest at a rate $\alpha_{2} N x_{2}$ : if there are $N x_{2}$ transporters within a time unit $\left(x_{2}\right.$ is the fraction of transporters and $N$ the number of potential hunters, so that $N x_{2}$ is the total number of transporters), each successfully transporting $\alpha_{2}$ dead bodies to the nest within that time unit, a total of $\alpha_{2} N x_{2}$ dead bodies are successfully carried to the nest during that time unit.

\section{Estimating Parameter Values}

In order to validate the model, we now have to check that the response functions follow the functional form assumed in the model, and the values of several of the model's parameters, $c_{1}$, $\theta_{1}, \tau_{1}, \alpha_{1}, c_{2}, \theta_{2}, \tau_{2}$, and $\alpha_{2}$, have to be estimated. The transition probabilities to and from the stinger and transporter states were measured as a function of the number of live prey, denoted by $n_{1}$, and the number of dead prey, denoted by $n_{2}$. Let $P_{i j}\left(n_{k}\right)$ be the probability per time unit that a worker exhibits a transition from behavioural state $i$ to behavioural state $j$ as a function of $n_{k}$ $(k=1,2)$, with the following conventions: $i=0$ corresponds to a non-hunter state, $i=1$ to the stinger state, and $i=2$ to the transporter state. These transition probabilities were measured only for those $N$ workers that exhibited a hunting behaviour (stinging or transporting) at least once during the course of an experiment. For example, $P_{01}\left(n_{1}\right)$ was measured as follows: for each individual that exhibited a hunting behaviour at least once during the experiment, we counted as to how often a transition from a non-hunter state to the stinger state was observed as a function of the value of $n_{1}$ at the time when the transition was observed. Our assumption is that transition probabilities to and from the two hunter states depend primarily on the levels of the stimuli associated with these hunter states, represented by $n_{1}$ and $n_{2}$. This, obviously, is a simplifying assumption that does not take into account the physiological state of the colony, possible recruitment mechanisms and additional stimuli.

Figures 3 and 4 show $P_{01}\left(n_{1}\right)$, the average probability of transition from state 0 to state 1 per minute per individual as a function of $n_{1}$, and $P_{02}\left(n_{2}\right)$, the average probability of transition from state 0 to state 2 per minute per individual as a function of $n_{2}$. Error bars along the $y$-axis correspond to averaging over individuals and experiments (in that order), and error bars along the $x$-axis correspond to averaging over data bins (see figure legends for more details). The dotted lines represent the best fits of the type $c_{i}\left(1-10^{-n_{i} / \theta_{i}}\right)$, where $\theta_{i}$ is a threshold and $c_{i}$ is a transition rate. One hypothesis that might explain why this type of functional form is observed is, roughly, the following: if, at each encounter with a live prey item, a worker has a fixed probability $\rho$ of responding to the item, then the probability that the worker will not respond to the first $n_{1}$ encountered items is given by $(1-\rho)^{n_{1}}$, and the probability that it will respond within the $n_{1}$ encounters is given by $1-(1-\rho)^{n_{1}}=1-10^{n_{1} \log (1-\rho)}=1-10^{-n_{1} / \theta_{1}}$ with $\theta_{1}=-1 / \log (1-\rho)$. This hypothesis, which has been introduced by Chrétien (1996) to explain the formation of cemeteries in the ant Lasius niger, remains to be tested for the present situation. For $P_{01}\left(n_{1}\right)$, we find $c_{1}=0.0553 \mathrm{~min}^{-1}$ -1 and $\theta_{1}=109.7$. For $P_{02}\left(n_{2}\right)$, we find $c_{2}=0.0386 \mathrm{~min}^{-1}$ and $\theta_{2}=15.9$. Notice that the dynamics of $n_{1}$ and $n_{2}$ are obviously correlated in these experiments, while we are assuming that they are independent: measuring the exact transition frequencies would require a 


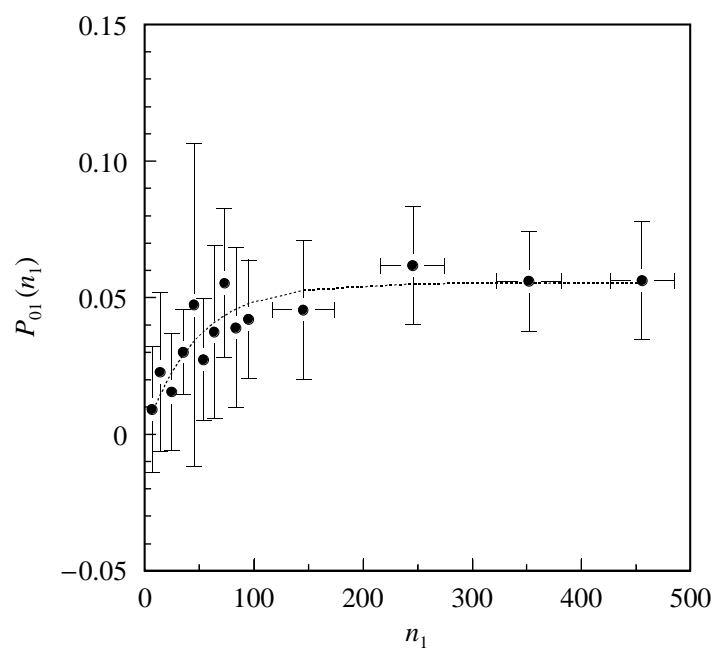

FIG. 3. Average probability of transition $P_{01}\left(n_{1}\right)$ from a non-hunter state to the stinger state per minute per individual as a function of $n_{1}$, the number of live prey in the hunting area. Error bars along the $y$-axis correspond to averaging over individuals and experiments (in that order), and error bars along the $x$-axis correspond to averaging over data bins. The following bins were used for live prey: 0-9, 10-19, 20-29, 30-39, 40-49, 50-59, 60-69, 70-79, 8089 , 90-99, 100-199, 200-299, 300-399, and 400-499. $(\ldots . .$.$) line represents best fit of the type$ $c_{1}\left(1-10^{-n_{1} / \theta_{1}}\right)$, with $c_{1}=0.0553 \mathrm{~min}^{-1}$ and $\theta_{1}=109.7$.

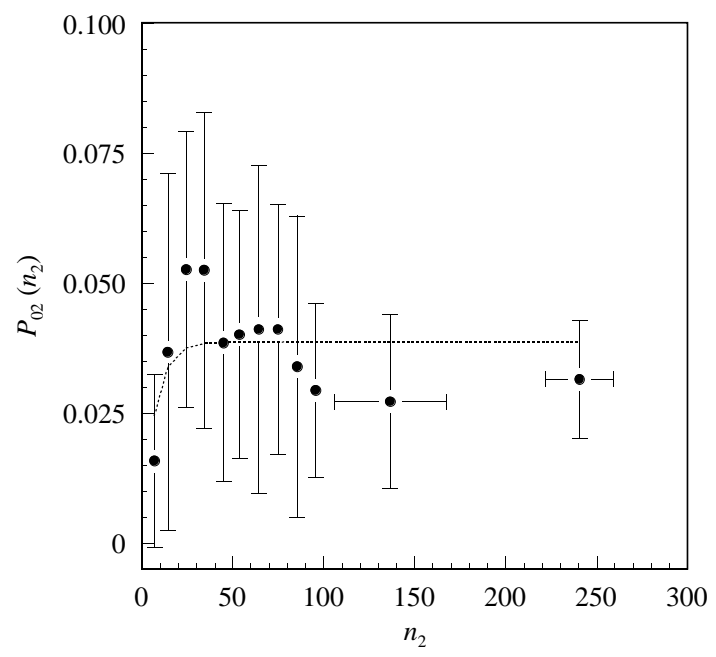

FIG. 4. Average probability of transition $P_{02}\left(n_{2}\right)$ from a non-hunter state to the transporter state per minute per individual as a function of $n_{2}$, the number of dead prey in the hunting area. Error bars along the $y$-axis correspond to averaging over individuals and experiments (in that order), and error bars along the $x$-axis correspond to averaging over data bins. The following bins were used for dead prey: $0-9,10-19,20-29,30-39,40-49,50-59,60-69,70-79,80-$ 89, 90-99, 100-199, and 200-299. (.....) line represents best fit of the type $c_{2}\left(1-10^{-n_{2} / \theta_{2}}\right)$, with $c_{2}=0.0386 \mathrm{~min}^{-1}$ and $\theta_{2}=15.9$. separate set of experiments with live prey only or dead prey only.

Individuals tended to specialize on either stinging or transporting within experimental trials. Transitions between stinging and transporting were rare. All other transition probabilities are either very small or do not depend significantly on $n_{1}$ and $n_{2}: P_{10}\left(n_{1}\right), P_{10}\left(n_{2}\right), P_{20}\left(n_{1}\right)$, and $P_{20}\left(n_{2}\right)$ can be considered constant with respect to $n_{1}$ and $n_{2}$, and $P_{12}\left(n_{1}\right), P_{12}\left(n_{2}\right), P_{21}\left(n_{1}\right)$, and $P_{21}\left(n_{2}\right)$ are all less than or equal to $0.008 \mathrm{~min}^{-1}$ for any value of $n_{1}$ or $n_{2}$, which means that the corresponding transitions are almost never observed during the experiments and can be neglected as a first approximation. Thus, we can define the probabilities of transition $P_{10}$ and $P_{20}$, or alternatively the average times $\tau_{1}=P_{10}^{-1}$ and $\tau_{2}=P_{20}^{-1}$ spent, respectively, in the stinger and transporter states before making a transition to a non-hunter state. We find that $\tau_{1}=33.2 \mathrm{~min}$ and $\tau_{2}=48.1 \mathrm{~min}$. Note that workers seem to spend on an average a relatively long time performing a given task, which certainly influences the observed colony-level dynamics, as do response thresholds. The large values of $\tau_{1}$ and $\tau_{2}$ might result from the fact that behavioural acts are recorded only every $10 \mathrm{~min}$ : more transitions could take place within those $10 \mathrm{~min}$. We also measured the efficiency of stinging, that is, the number of live prey killed per stinger per time unit, denoted by $\alpha_{1}$, and the efficiency of transporting, that is, the number of corpses successfully transported to the nest per transporter per time unit, denoted by $\alpha_{2}$. The average over all the experiments yields: $\alpha_{1}=0.33 \mathrm{~min}^{-1}$ and $\alpha_{2}=0.22 \mathrm{~min}^{-1}$.

\section{Results}

Equations (1)-(4) cannot be solved analytically, except in special cases. Equations (1)-(4) were integrated numerically, using the estimated values of the parameters, in three distinct conditions, which correspond to the three experimental situations described in Section 2:

(i) 130 workers $(S=130, N=12), 80$ live prey $\left(n_{1}(t=0)=80\right)$.

(ii) 130 workers $(S=130, N=15), 250$ live prey $\left(n_{1}(t=0)=250\right)$. 
(iii) 240 workers $(S=240, N=22), 500$ live prey $\left(n_{1}(t=0)=500\right)$.

In all the three cases, the initial conditions are: $x_{1}(t=0)=x_{2}(t=0)=0$ (no worker is in a hunting state) and $n_{2}(t=0)=0$ (there are initially no corpses in the hunting area). Figure 5 shows the results of the numerical integration and it has to be compared to Fig. 1. In all the three cases, there is a striking similarity between the dynamical patterns observed during the experiments and the dynamical patterns obtained with the model. These different patterns arise in both the experiments and the model by changing the numbers of workers and hunters and/or the number of live prey initially available in the hunting area. The quantitative differences observed between the experiments and the model are likely to result from the approximations of the model (for example, assuming that $n_{1}$ and $n_{2}$ are uncorrelated to estimate transition probabilities, assuming that transition probabilities depend only on $n_{1}$ and $n_{2}$, averaging over all workers, neglecting recruitment, inaccuracy of some of the estimates, etc.).

\section{Discussion}

Task partitioning occurs in many species of social insects. It defines an important and apparently widespread feature of work organization in social insects. The study of different examples reveals that this feature has actually evolved several times (Ratnieks \& Anderson, 1999). Together with an appropriate identification of its presence in a given system, a quantitative characterization of the intrinsic dynamics is needed. Previous studies involving
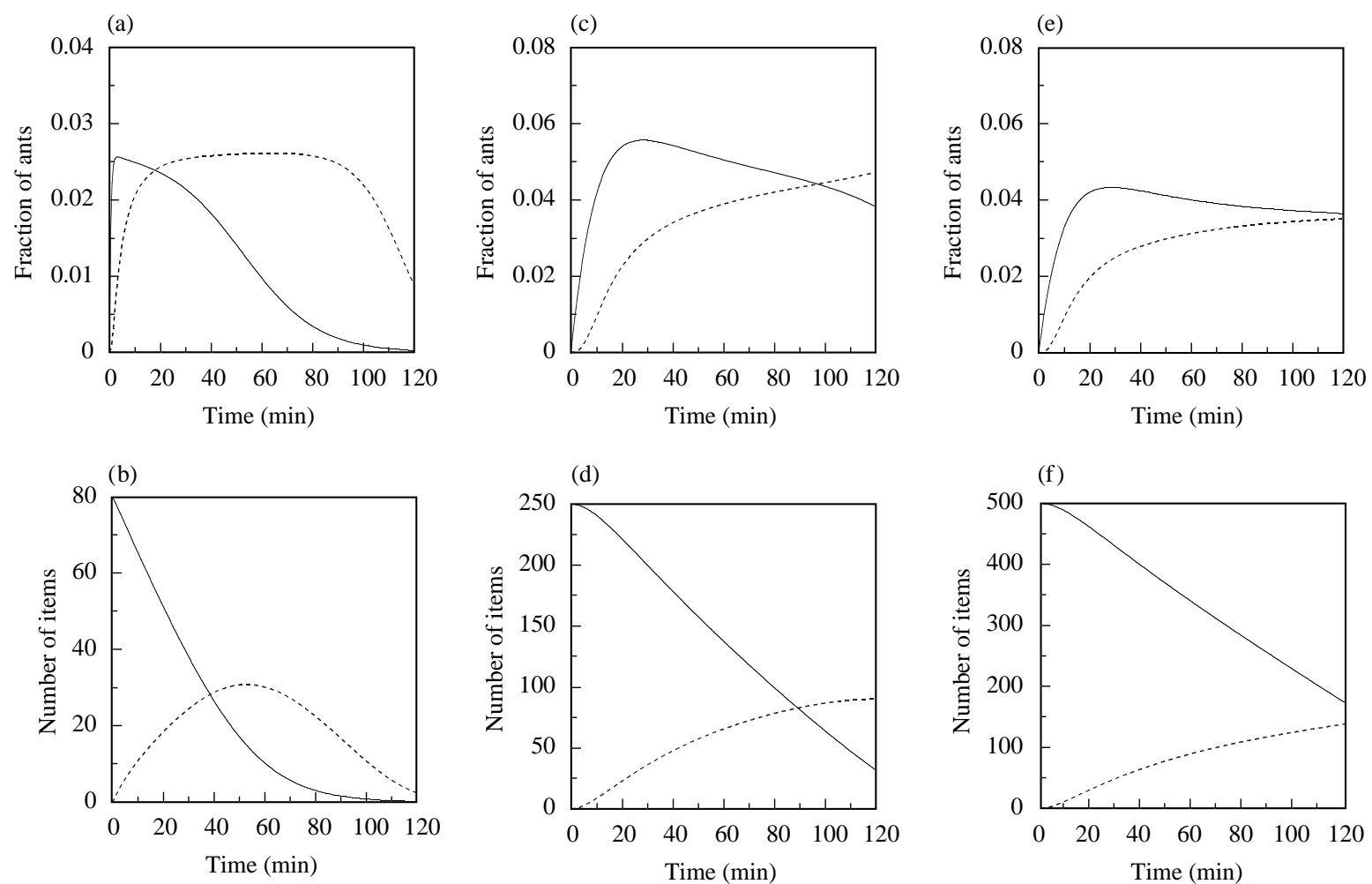

FIG. 5. Fractions of colony workers that are stingers $\left(f_{1}\right)$ or transporters $\left(f_{2}\right)$, and numbers of live $\left(n_{1}\right)$ and dead $\left(n_{2}\right)$ prey in the hunting area as a function of time obtained from numerical integration of eqns (1)-(4) for three different colony sizes and initial number of prey $(\mathrm{a}, \mathrm{b})$. The initial number of prey is 80 and the number of workers is 130 (parameters: $S=130, N=12, x_{1}(t=0)=x_{2}(t=0)=0, n_{1}(t=0)=80$ and $\left.n_{2}(t=0)=0\right)(\mathrm{c}, \mathrm{d})$. The initial number of prey is 250 and the number of workers is 130 (parameters: $S=130, N=15, x_{1}(t=0)=x_{2}(t=0)=0, n_{1}(t=0)=250$ and $\left.n_{2}(t=0)=0\right)(\mathrm{e}, \mathrm{f})$. The initial number of prey is 500 and the number of workers is 240 (parameters: $S=240, N=22, x_{1}(t=0)=x_{2}(t=0)=0$, $n_{1}(t=0)=500$ and $\left.n_{2}(t=0)=0\right)$. For all the three conditions: $\alpha_{1}=0.33 \mathrm{~min}^{-1}, \alpha_{2}=0.22 \mathrm{~min}^{-1}, \theta_{1}=109.7$ live prey, $\theta_{2}=15.9$ dead prey, $c_{1}=0.0553 \mathrm{~min}^{-1}, c_{2}=0.0386 \mathrm{~min}^{-1}, \tau_{1}=33.2 \mathrm{~min}$, and $\tau_{2}=48.1 \mathrm{~min}$. (a, c, e) $(-) f_{1} ;(\cdots \cdots) f_{2} .(\mathrm{b}, \mathrm{d}, \mathrm{f})$ $(-) n_{1} ;(----) n_{2}$. 
division of labour in the ants Pheidole successfully reproduced the main quantitative features displayed by experiments (Bonabeau et al., 1996). This study used a threshold model that has been extended in our study to the analysis of a well-defined example of task partitioning.

The response threshold model described in this paper is simple, plausible, consistent with experiments, relies solely on empirically measured parameter values and reproduces nontrivial dynamical colony-level patterns with a minimum of assumptions. Very few models of division of labour in social insects have currently been tested with experimental data (Beshers \& Fewel, 2001). Here, we show that the combination of modelling and empirical work connects explicitly individual behaviour to colony-level behaviour through a set of response thresholds, the existence of which has been shown. This quantitative example clearly shows that a difference in the response thresholds associated with the two kinds of stimuli (live prey and corpses) is a sufficient condition to generate task partitioning among workers. It also shows how the interplay between the dynamics of the phenomenon combined with individuals' response thresholds affects colony-level patterns. This work suggests a new methodology to study task partitioning in social insects; it emphasizes the importance of designing new experiments to study how individuals' behavioural responses change with the value of the stimuli associated with the tasks.

The next step will be to take interindividual differences into account in the model. In its current form, the model provides an average description of the hunting pattern. Averaging out interindividual differences is fine if one wishes to reproduce global patterns, but a degree of specialization among hunters has been reported (Schatz et al., 1996). This specialization suggests, within the response threshold framework, innate threshold differences between workers and/or learning or habituation (Theraulaz et al., 1998). A model combining interindividual differences and learning (Theraulaz et al., 1998) could explain the transition from no task partitioning (hunters sting and transport) to task partitioning (stinging and transporting are usually performed by two distinct workers) as the size of the group of hunters increases and on a longer time scale. A similar phenomenon was observed in the eusocial wasp species Polybia occidentalis (Jeanne, 1991) and Mischocyttarus mastigophorus (O'Donnell, 1998). This points to the role of colony size as an important evolutionary parameter (Karsai \& Wenzel, 1998; Bourke, 1999; Anderson \& McShea, 2001), the impact of which could be better understood with a threshold model.

This work was supported in part by a grant from the GIS (Groupement d'Intérêt Scientifique) Sciences de la Cognition to E. B and G. T. E. B. is supported by the Interval Research fellowship at the Santa $\mathrm{Fe}$ Institute, G. T. by a grant from the Conseil Régional Midi-Pyrénées and B. S. by a grant Sciences de la Cognition (MESR). We wish to thank Guy Beugnon and Jean-Paul Lachaud for their help and advice.

\section{REFERENCES}

Agbogba, C. \& Howse, P. E. (1992). Division of labour between foraging workers of the ponerine ant Pachycondyla caffraria (Smith) (Hymenoptera: Formicidae). Insectes Soc. 39, 455-458.

Anderson, C. \& McShea, D. W. (2001). Individual versus social complexity, with particular reference to ant colonies. Biol. Rev. (Camb.) 76, 211-237.

Anderson, C., Franks, N. R. \& Mcshea, D.W. (2001). The complexity and hierarchical structure of tasks in insect societies. Anim. Behav. 62, 643-651.

Besher, S. N. \& Fewell, J. H., (2001). Models of division of labor in social insects. Ann. Rev. Entomol. 46, 413-440.

Bonabeau, E., Theraulaz, G. \& Deneubourg, J.-L. (1996). Quantitative study of the fixed threshold model for the regulation of division of labour in insect societies. Proc. Roy. Soc. London B 263, 1565-1569.

Bonabeau, E., Theraulaz, G. \& Deneubourg, J.-L. (1998). Fixed response thresholds and the regulation of division of labour in social insects. Bull. Math. Biol. 60, 753-807.

Bourke, A. F. G. (1999). Colony size, social complexity and reproductive conflict in social insects. J. Evol. Biol. 12, 245-257.

Bourke, A. F. G. \& Franks, N. R. (1995). Social Evolution in Ants. Princeton, NJ: Princeton University Press.

Chrétien, L. (1996). Organisation spatiale du matériel provenant de l'excavation du nid chez Messor barbarus et des cadavres d'ouvrières chez Lasius niger (Hymenopterae: Formicidae). Ph.D. Dissertation, Département de Biologie Animale, Université Libre de Bruxelles.

JEANNe, R. L. (1986). The evolution of the organization of work in social insects. Monitor Zool. Ital. 20, 119-134.

Jeanne, R. L. (1991). Polyethism. In: The Social Biology of Wasps (Ross, K. G. \& Matthews, R. W., eds), pp. 389-425. Ithaca, NY: Cornell University Press. 
Karsai, I. \& Wenzel, J. W. (1998). Productivity, individual-level and colony-level flexibility, and organization of work as consequences of colony size. Proc. Natl Acad. Sci. U.S.A. 95, 8665-8669.

LACHAUD, J.-P. (1990). Foraging activity and diet in some neotropical ponerine ants. I. Ectatomma ruidum Roger (Hymenoptera, Formicidae). Folia Entomol. Mex. 78, 241-256.

O'Donnell, S. (1998). Dominance and polyethism in the eusocial wasp Mischocyttarus mastigophorus (Hymenoptera: Vespidae). Behav. Ecol. Sociobiol. 43, 327-331.

Oster, G. \& Wilson, E. O. (1978). Caste and Ecology in the Social Insects. Princeton, NJ: Princeton University Press.

Plowright, R. C. \& Plowright, C. M. S. (1988). Elitism in social insects: a positive feedback model. In: Interindividual Behavioral Variability in Social Insects (Jeanne, R. L., ed.), pp. 419-431. Boulder, CO: Westview Press.

Ratnieks, F. L. W. \& Anderson, C. (1999). Task partitioning in insect societies. Insectes Soc. 46, 95-108.
Robinson, G. E. (1987). Modulation of alarm pheromone perception in the honey bee: evidence for division of labor based on hormonally regulated response thresholds. J. Comp. Physiol. A 160, 613-619.

Robinson, G. E. (1992). Regulation of division of labour in insect societies. Ann. Rev. Entomol. 37, 637-665.

Schatz, B. (1997). Modalités de la recherche et de la récolte alimentaire chez la fourmi Ectatomma ruidum Roger: flexibilités individuelle et collective. Ph.D. Dissertation, Université Paul Sabatier, Toulouse, France.

Schatz, B., Lachaud, J.-P. \& Beugnon, G. (1996). Polyethism within hunters of the ponerine ant, Ectatomma ruidum Roger (Formicidae, Ponerinae). Insectes Soc. 43, 111-118.

Schatz, B., Lachaud, J.-P. \& Beugnon, G. (1997). Graded recruitment and hunting strategies linked to prey weight in the neotropical ant, Ectatomma ruidum Roger. Behav. Ecol. Sociobiol. 40, 337-349.

Theraulaz, G., Bonabeau, E. \& Deneubourg, J.-L. (1998). Response threshold reinforcement and division of labour in insect societies. Proc. Roy. Soc. London B 265, $327-332$. 